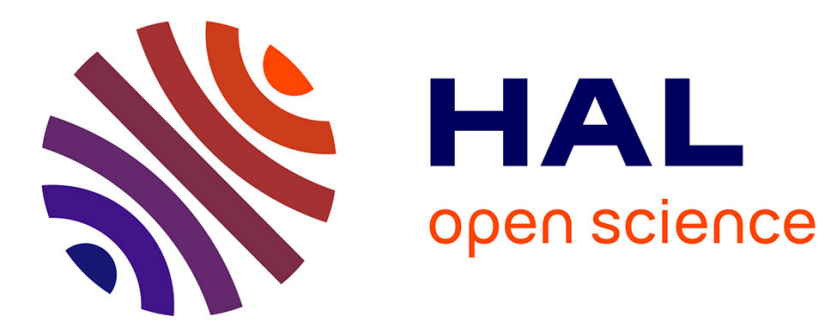

\title{
Skyline-based Approach for Natural Scene Identification
}

Ameni Sassi, Chokri Ben-Amar, Serge Miguet

\section{To cite this version:}

Ameni Sassi, Chokri Ben-Amar, Serge Miguet. Skyline-based Approach for Natural Scene Identification. 13th ACS/IEEE International Conference on Computer Systems and Applications (AICCSA 2016), Nov 2016, Agadir, Morocco. hal-01385537

\section{HAL Id: hal-01385537 \\ https://hal.science/hal-01385537}

Submitted on 21 Oct 2016

HAL is a multi-disciplinary open access archive for the deposit and dissemination of scientific research documents, whether they are published or not. The documents may come from teaching and research institutions in France or abroad, or from public or private research centers.
L'archive ouverte pluridisciplinaire HAL, est destinée au dépôt et à la diffusion de documents scientifiques de niveau recherche, publiés ou non, émanant des établissements d'enseignement et de recherche français ou étrangers, des laboratoires publics ou privés. 


\section{Skyline-based Approach for Natural Scene Identification}

\author{
Ameni Sassi, Chokri Ben Amar \\ REGIM-Lab.: REsearch Groups in Intelligent Machines \\ University of Sfax, ENIS, BP 1173, 3038, \\ Sfax, Tunisia \\ \{ameni.sessi.tn, chokri.benamar\}@ieee.org
}

\author{
Serge Miguet \\ LIRIS, Université de Lyon, UMR CNRS 5202, \\ Université Lumière Lyon 2, 5 av. Mendès-France, Bât C, \\ $\mathrm{N}^{\circ} 123,69676$. Bron, Lyon ,France \\ serge.miguet@univ-lyon2.fr
}

\begin{abstract}
The skyline, defined as the line separating the sky from other objects on the ground, could provide unique and useful information for a variety of applications. This line was used as a key data, especially, for geo-localization and aerial robotic applications. The particular shape and the geometric features of a skyline may be the identity of the landscape itself. The skyline, once well extracted, could show the silhouette of a famous tower, the mountain peaks, or the landscape topography. In this paper, we proposed a geometric description of the extracted skyline from landscapes. Based on some geometric descriptors, we tried to pick up practical measurements for each skyline. The first proposed approach was the straight lines' classification to differentiate between urban and natural landscapes from their horizon line. The second one is the curvature analysis using a Curvature Scale Space descriptor. This descriptor was used to enhance the first one and to distinguish between natural part and buildings in the same skyline. The results obtained from these geometric description tools were very competitive and they will be the inputs for a classification process.
\end{abstract}

Keywords—skyline; geometric descriptor; landscape; curvature

\section{INTRODUCTION}

Watching a cityscape from high rise, we can notice that besides the indentation created by tall buildings, the "nature" elements such as vegetation and topographical context may increase the complexity of urban landscape representations. The appearance of these natural elements in a cityscape could affect the reviews of citizens or visitors about that landscape. Our distant goal is to objectify the influence of natural elements on the representations of urban landscapes using a photo-questionnaire method. This was in the context of a sustainable city project that aims to identify and systematically analyze the skyline perceptions of practitioners and the general public by matching the characteristics taken from skyline photographs with the perceptions of several audiences in some European cities (Lyon and London). The first characteristic identified and extracted from landscapes was the skyline, defined as the line that marks the boundary between the sky and the ground elements (natural or artificial). Based on the obtained skyline, we will try to extract other useful measurements to describe that line and use it for our purposes. Our first goal is the identification of natural elements within the skyline. In fact, this line can give a preliminary idea about the existing elements in a landscape. We can, for example, know that there are tall buildings or large mountains by scanning the skyline of a specific photography. The Fig.1 shows the skyline of Chicago where we can easily discriminate the towers and the tall buildings. Because of the unique aspects that could contain a skyline, we have chosen it to be the first tool to interpret the images of landscapes.

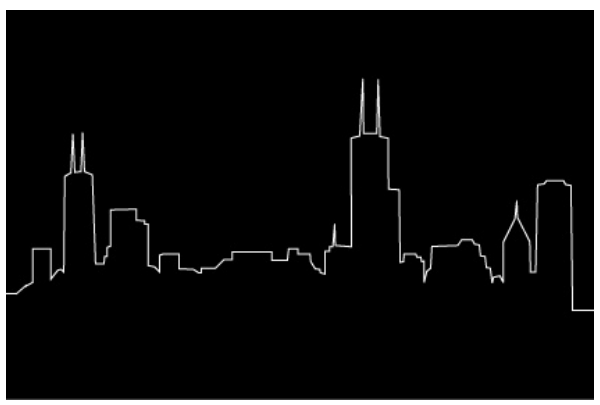

Fig. 1. Chicago's Skyline

This paper is mainly divided into four parts. The first part reveals some related works about how the skyline was used for different applications. Then, in the second part, we will describe the geometric description tools used to analyze the skyline of landscapes. After that we present some first results in the third section. The last section of this paper contains the conclusions and some perspectives that will be the goal of a future work.

\section{RELATED WORKS}

The fact that the horizon line is highly informative was proved in the literature by using the information extracted from skylines for different purposes, such as navigation in airborne [1][2][4] and marine vehicles [3], visual geo-localization [5][6][7] and also annotation of landscapes [8][9].

For vision-based navigation, many researchers [1][2][3] suggested to use the skyline as a key information, instead of the GPS and the INS, to estimate the position and the orientation of the Unmanned Arial Vehicles UAV. Using vision-based methods to extract the skyline and some useful points on it, and then matching the extracted data with a Digital Elevation Map (DEM) could be the only required steps to estimate accurately the position and the attitude of the UAV camera. The challenge in these methods is to extract the skyline and the appropriate 
features under noisy images, bad weather and variable luminosity conditions. Reference [2] proposed a vision-based navigation system to estimate the localization of UAV for a mountain-region use. The purpose was to accurately extract the skyline and the position of some key peaks on the mountain from infrared images. The use of IR (infrared) images was a solution for some challenges, but the narrow field of view of traditional cameras restricts the viewed part of the skyline. To deal with this problem, the authors in [1] proposed a challenging algorithm to extract the skyline in catadioptric infrared images. Compared to traditional infrared cameras, the catadioptric IR systems could cover a much larger part of the horizon scene which improve the matching with the Digital Elevation Map. Another type of images was used in [4], that proposed a sky segmentation using panoramic ultraviolet UV images.

Since the skyline could provide robust and unique features, we can recognize the area where a photo was taken by looking only at the skyline. So, extracting the skylines and matching them to a database of known skyline shapes or digital elevation maps could be an effective tool for automated geo-localization of images. The geo-localization can be applied for urban landscapes [5] or also for natural ones [6] [7]. Reference [5] relies only on a skyline extraction algorithm from omnidirectional images for an accurate geo-localization within the streets of a city. The extracted skyline is matched to the ones extracted from 3D city models to estimate the position of the omnidirectional camera. Another research work on urban geolocalization [6], proposed to use the building skyline, some useful points on it and the skylines pre-rendered from digital elevation maps, to estimate the camera position. The Digital Elevation Models and 3D models have shown promising results for the geo-localization in natural landscapes as well. In this work [7], the authors have proposed a work for geolocating queries in a large scale mountainous terrain by matching against skylines pre-rendered from digital elevation models. These works of vision-based geo-localization are almost useful when the GPS information is not available and they could be helpful for geo-tagging applications. In this geotagging application [8], they have proposed an automatic extraction algorithm of the skyline to match it to a database of reference skylines in order to geo-tag photos and images in natural terrain. The extracted skyline was used here [9] to annotate the positions and names of mountains peaks.

As proved in the previously-mentioned research works, the skyline could be the key data for a variety of applications. The way of operating this line depends on the requirements of each application. In our work, the main aspects used to interpret the skyline were the specific geometric features that differ from an horizon line to another.

\section{SKYLINE'S GEOMETRIC DESCRIPTION}

Based on the extracted skyline, we tried to take out useful measurements to distinguish between urban landscape photos and natural landscape photos and thereafter the natural part and the buildings one in the same landscape. As we can notice in the Fig. 2, buildings and towers are generally represented by regular geometric forms and straight segments, unlike the natural elements that tend to occur more irregular. The buildings are mostly represented by long segments, whereas the natural skyline part is often composed by many short segments. If we define the corner as the intersection between straight segments, then the number of corners is not the same in the two parts. Moreover, the curvature seems to have dissimilar values all along the skyline; which could be a way to detect some key points on the skyline.

The skyline was seen as an open curve or a polyline. Thus, some geometric descriptors could be applied to reach our goal. The first geometric description tool was based on the length of straight segments. The second geometric descriptor was the Curvature Scale Space representation to analyze the horizon line point per point.

\section{A. The straight lines classification}

As we have mentioned before, the buildings and towers in landscapes are characterized by long straight lines, especially when there is a high tower. So, the idea was to break the skyline into segments through a polygonal approximation algorithm. The skyline is, then, approximated into a fewer number of vertices and straight segments depending on a precision parameter. The value given to this parameter must preserve the small details of the approximated skyline; it is supposed to eliminate the noise and obtain truly straight segments. The next step was to classify the straight lines, obtained from the polygonal approximation, according to their length. The result of this process is a histogram that presents the number of segments on each category of segments length. To avoid getting categories with zero segments and to have an appropriate histogram to each skyline and landscape photography, we thought about making variable categories' intervals. The limits of intervals follow a pattern of geometric sequence. The width of the interval of each category depends on three elements: (i) the number of categories itself categ, (ii) the shortest segment $l$ in the approximated skyline and (iii) the height of the landscape image. The equations $(1 \rightarrow 4)$ explain better the choice of categories limits.

$$
\begin{aligned}
& \text { interval }_{1}=[0, l] \\
& \text { interval }_{2}=[l, l+l x] \\
& \text { interval }_{3}=\left[l+l x, l+l x+l x^{2}\right] \\
& \text { interval }_{\text {categ }}=\left[l\left(\frac{1-x^{\text {categ-1 }}}{1-x}\right), l\left(\frac{1-x^{\text {categ }}}{1-x}\right)\right] \\
& \text { where, } x=\left(\frac{L}{l}\right)^{\frac{1}{\text { categ-1 }}} \text { and, } L=\frac{\text { image_Height }}{\text { categ }}
\end{aligned}
$$

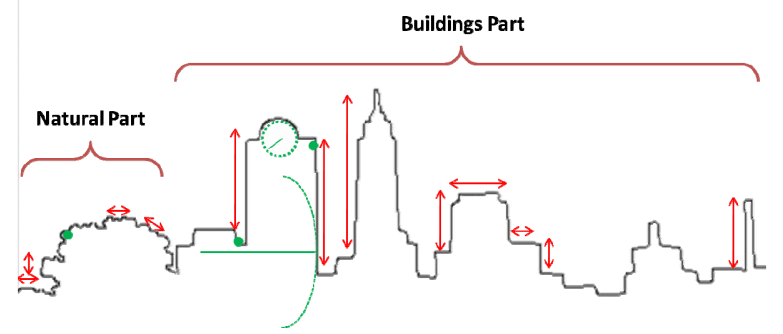

Fig. 2. Skyline's parts 
The comparison between the number of straight lines in each category for different skylines, could be a way to differ urban landscapes from natural ones. Nevertheless, this manner of comparison would not be adequate since the width of each category is not the same for all landscapes. Therefore, the idea of classifying straight lines using histograms was manipulated to obtain an indicative value of segments' length for each skyline. This step will be better explained in section IV-A while showing the results.

\section{B. The curvature analysis}

Looking at a skyline, we can affirm that the degree of curvature goes through a lot of changes along this line. It is obvious that the indentation created by a tree, the mountain peaks and the structure of a building seem to have different values of curvature. The formula to calculate the curvature, we admitted, is (5) where the skyline is defined as the curve (6). The representation of the curvature along the skyline gives a graphic as shown in the Fig. 3; the curvature value could be positive, negative or null. The sudden changes in these values label the key points on the skyline.

$$
\begin{gathered}
k(t)=\frac{\dot{x}(t) \ddot{y}(t)-\ddot{x}(t) \dot{y}(t)}{\left(\dot{x}^{2}(t)+\dot{y}^{2}(t)\right)^{3 / 2}} \\
\vec{\tau}(t)=(x(t), y(t))
\end{gathered}
$$

To better discover the geometric aspects along the skyline and select the persistent key points, we have proceeded to the curvature scale-space description. The CSS (Curvature Scale Space) descriptor was introduced by F. Mokhtarian and A.K. Mackworth [10] and it can be used for shape similarity retrieval [11] and also for corner detection [12]. The process consists on computing the curvature values for a curve that have to be smoothed gradually using Gaussian kernels that precise the scale. So, at each scale we have different values of curvatures corresponding to the smoothed curve. As we have said before, the curvature goes through negative values and positive ones. The zero-crossings of curvature at different scales are the way to find the concavities/convexities of a curve which are helpful to find some perceptual features within this curve. Fig. 4 explains the output of the CSS descriptor for a closed contour. The CSS map illustrates the lifetime of the curve's points all over the increasing scales. The zero-crossings of the curvature could be picked from the CSS image for each scale.

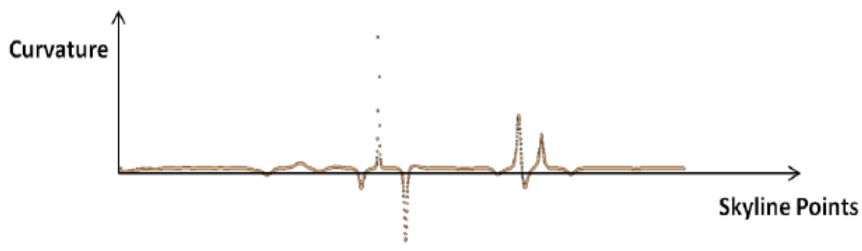

Fig. 3. The curvature function

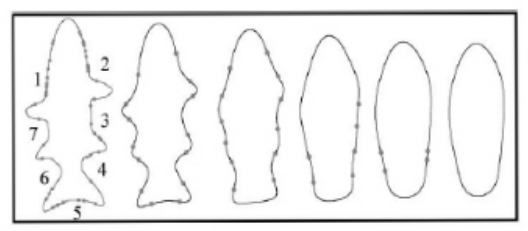

(a)

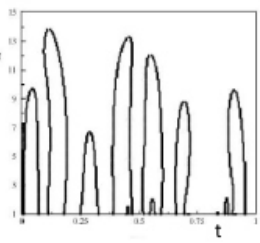

(b)
Fig. 4. (a) The smoothing process of the curve showing the number of zerocrossings curvature for the scale $=1,4,7,10,12,14$ and (b) the CSS map [11]

The application of the CSS descriptor to the skyline gives important information that could be practical to take out the geometric features of each part on the skyline. The manner how we analyze the curvature and the results obtained are illustrated in the section IV-B.

\section{EXPERIMENTAL RESULTS}

\section{A. Lengths histograms and segments 'mean size'}

The classification of straight lines composing the skyline is shown as histograms with variable categories as indicated before. The obtained histograms for two different landscapes are shown in Fig. 8. We can notice that the numbers of segments in each category vary from a landscape to another. For New York skyline, the histogram shows that there is a considerable number of long straight segments unlike the histogram generated for the natural landscape skyline that depicts a very small number of long segments and a very important number of short segments. To describe the skyline with a concrete value, we decided to generate other histograms with different distribution. The idea was to make a link between the number of segments for each length and the length itself, in order to highlight the relation between the number of segments and its length. The axes and the distribution of these histograms are more explained in the Fig. 5 and Fig. 6. The cumulated histograms, obtained from the histograms showing the number of segments per each segment size multiplied by the size, give an idea about the ' mean size' of segments. We expect to have a higher value for urban landscapes than natural ones. The mean segments size, derived from the cumulated histogram, could be an index for describing the skyline segments in a general way: the skyline is almost natural or artificial.

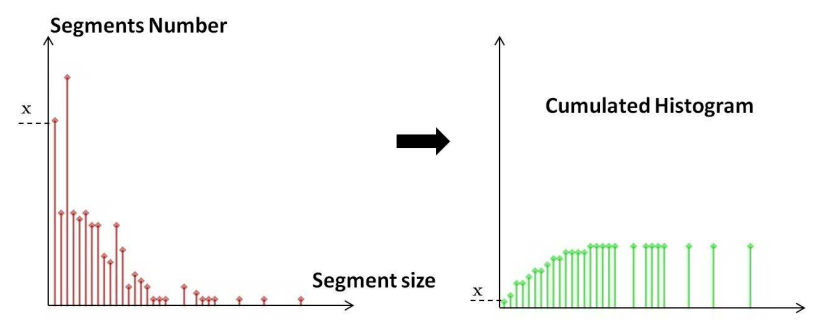

Fig. 5. Simple cumulated histogram 


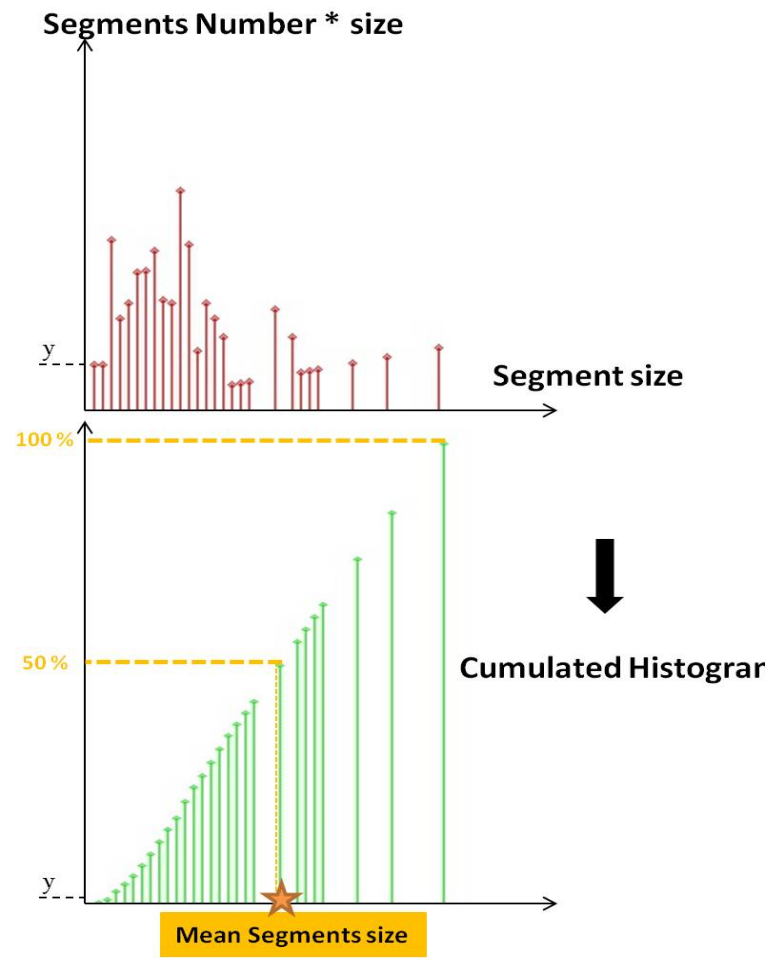

Fig. 6. Modified cumulated histogram

The mean segments sizes that we got for some landscapes are listed in Table 1. Observing these values, we can notice that urban landscapes are described by a high index of segments size whereas the natural ones show smaller values. This skyline segments classification was not crucial for some urban landscapes where the photo is taken from faraway or there are no tall buildings like the case of the photo of the Istanbul landscape (Fig. 7). Moreover, these histograms have given an idea about the entire skyline and could not help to specify which part represents a natural element and which one shows buildings.

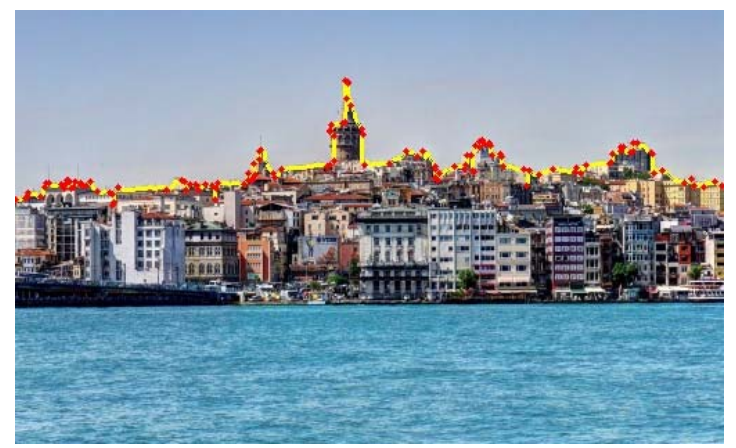

Fig. 7. The landscape of Istanbul

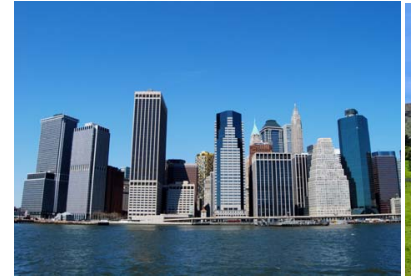

(a)

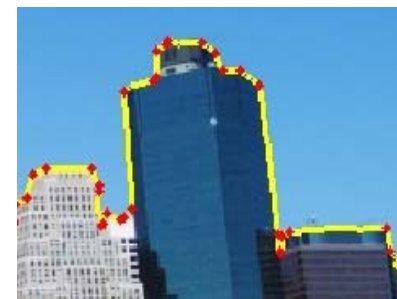

(c)

\section{Segments'}

4 number

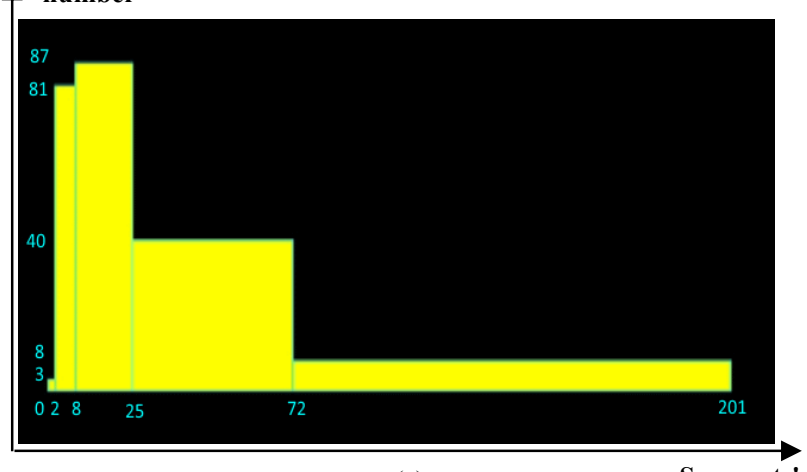

(e) Segments Length

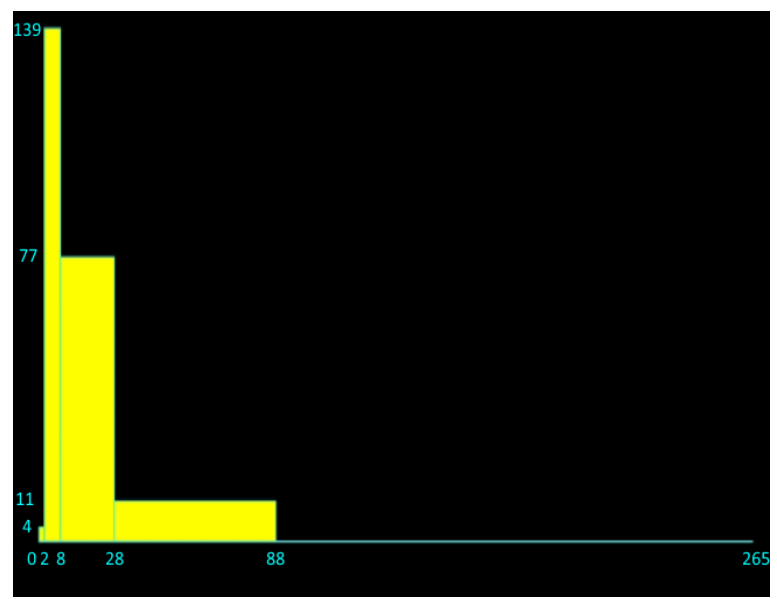

(f)

Fig. 8. Classification of the segments of the approximated skyline

(a) New York landscape (b) Natural landscape (c)(d) zoom on the result of polygonal approximation of the skyline (e)The classification histogram for New York landscape (f) The classification histogram for the natural landscape 5 
TABLE I. THE MEAN LENGTH INDEX FOR DIFFERENT LANDSCAPES

\begin{tabular}{|l|c|}
\hline \multicolumn{1}{|c|}{ Landscape } & $\begin{array}{c}\text { Index of the Mean } \\
\text { Segments size }\end{array}$ \\
\hline New York & 63 \\
\hline Boston & 61 \\
\hline Dubai & 55 \\
\hline Shanghai & 35 \\
\hline Chicago & 53 \\
\hline Madrid & 58 \\
\hline London & 28 \\
\hline Istanbul & 25 \\
\hline Natural Landscape2 & 22 \\
\hline Natural Landscape4 & 30 \\
\hline Natural Landscape5 & 32 \\
\hline Natural Landscape6 & 38 \\
\hline Natural Landscape8 & 21 \\
\hline Natural Landscape9 & 25 \\
\hline Vancouver & 32 \\
\hline
\end{tabular}

\section{B. The evolution of skyline key points based on curvature}

The first step of the CSS description algorithm is computing the CSS map, starting with re-sampling the skyline into a fixed number of points, to calculate the curvature for every point at different increasing scales. After that, the values of curvature are scanned at each scale to select the points where the curvature passes through zero. These points are called the zero-crossings points and they are the dots composing the CSS map. The Fig. 9 shows an example of a CSS map generated for a skyline where the blue curves represent the zero-crossing points of curvature over different scales of smoothing. It is clear from the figure that the number of zero-crossings at high scales is fewer than the ones found in low scales since the curve is very well smoothed in coarse scales and there will be few concavities and convexities.

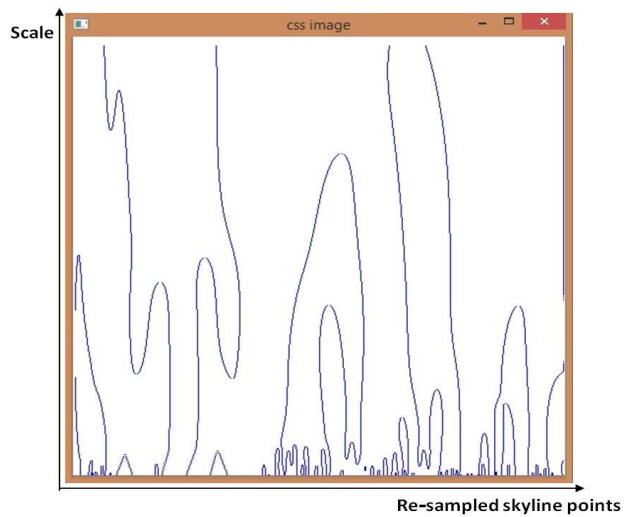

Fig. 9. The CSS image
Applying the curvature descriptor to our extracted skylines, we obtained interesting results. Fig. 11 (a)(b) depict a zoom-on parts of the colored skylines; the points where the curvature is almost constant are in yellow (straight lines) and the red or green points are the points where the skyline change the direction to a concavity point (Green) or a convexity one (Red). Observing the CSS map and the colorful skyline over different scales for two different landscapes (urban and natural), we can conclude that this descriptor was useful for tracking the evolution of key points on the skyline. The Fig. 11 shows a zoom-on the colored skyline for the New York landscape and the natural landscape at the scale 1 (a)(b) and the scale 5 (c)(d) behind their generated CSS map. What we can notice from these images is that there is an interesting number of key points (concavity and convexity) in the natural landscape at fine scale that disappear quickly by increasing the scale of smoothing (scale 5). In contrary, a visible number of inflexion points still appear at the same scale for the urban landscape. By comparing the skylines obtained in different scales for a variety of landscapes, we can notice that for the natural landscapes there was an interesting number of key points that reflect the fast variation in the curvature but these points disappeared fast from low scales. In contrast, the remarkable points in urban skylines, such as the buildings corners, resist until high scales. To translate the observations with concrete values, we chose to represent the waning of the number of key points across the different scales through scatter plots as shown in the Fig. 10. To describe these graphics using numbers, we calculated the percentage of key points that resist until a specific scale. The smoothing scale we have chosen to compute these rates was the middle of the scales' interval. To justify our observations, these percentages for cityscapes should be higher than the ones obtained for natural landscapes. The results for some landscapes of our test images are summarized in Table 2. The percentage of key points on the skyline of natural landscapes that resist until the middle scale seems to be lower than the percentage for urban landscapes; which prove that the small serration caused by natural elements on the skyline disappears from low scales of smoothing.

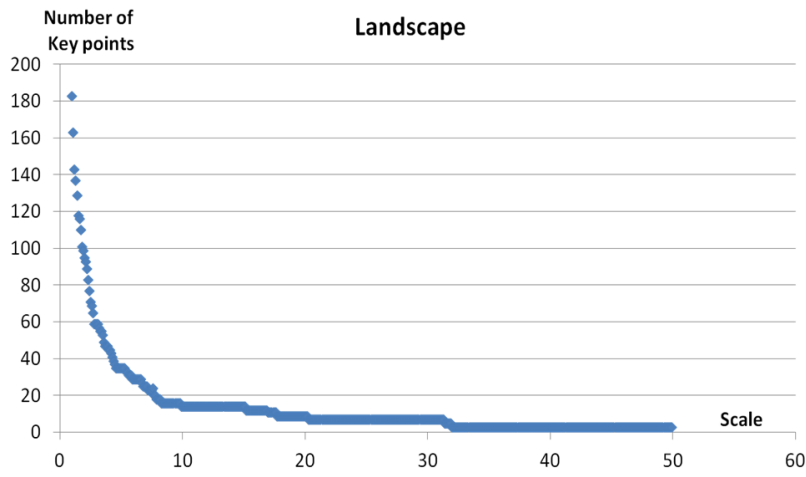

Fig. 10. The number of skyline key points over smoothing scales 

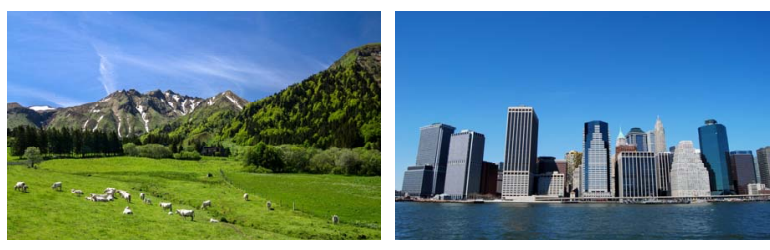

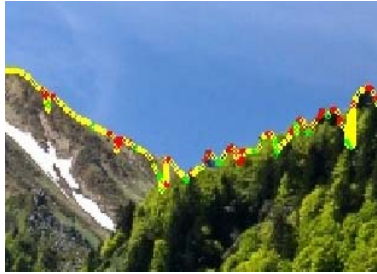

(a)

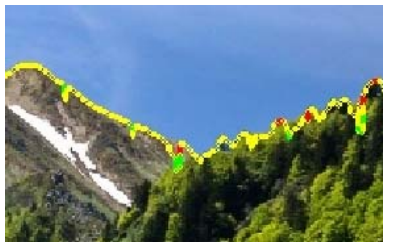

(c)

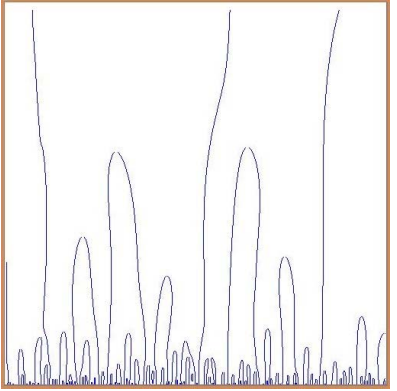

(e)

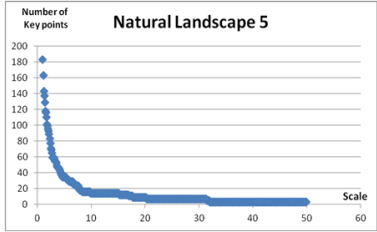

(g)

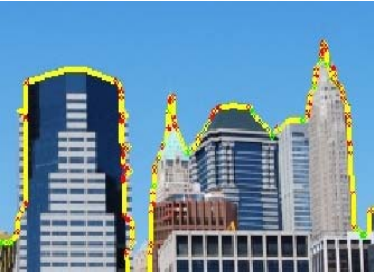

(b)

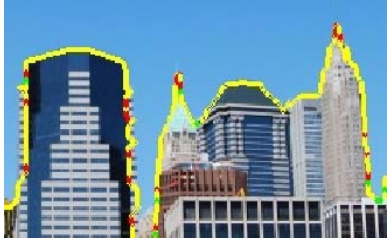

(d)

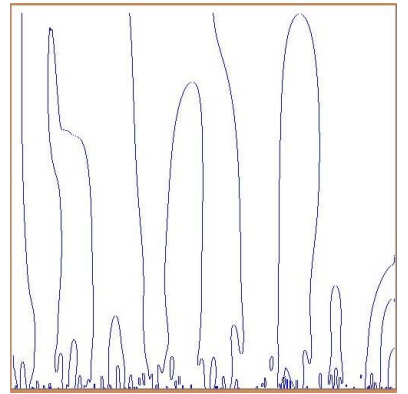

(f)

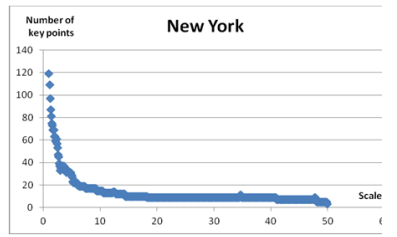

(h)
Fig. 11. (a)(b) Zoom on the Colorful skyline at the scale 1(c)(d) Zoom on the Colorful skyline at the scale 5 (e)(f) The CSS MAP (g)(h) The evolution of the number of skyline key points over the scales

TABLE II. THE PERCENTAGE OF RESISTING POINTS FOR DIFFERENT LANDSCAPES

\begin{tabular}{|l|c|}
\hline \multicolumn{1}{|c|}{ Landscape } & $\begin{array}{c}\text { \% Percentage of resisting key } \\
\text { points at the middle scale }\end{array}$ \\
\hline New York & 7.56 \\
\hline Boston & 11.68 \\
\hline Dubai & 6.36 \\
\hline Shanghai & 8.08 \\
\hline Chicago & 8.65 \\
\hline Madrid & 16.12 \\
\hline
\end{tabular}

\begin{tabular}{|l|c|}
\hline London & 6.83 \\
\hline Istanbul & 6.66 \\
\hline Natural Landscape2 & 3.63 \\
\hline Natural Landscape4 & 5.71 \\
\hline Natural Landscape5 & 3.82 \\
\hline Natural Landscape6 & 4.73 \\
\hline Natural Landscape8 & 5.69 \\
\hline Natural Landscape9 & 4.11 \\
\hline Vancouver & 11.2 \\
\hline
\end{tabular}

The images collected in the Fig. 12 show the results obtained for four different landscapes: Shanghai landscape, Chicago landscape and two natural landscapes. The histograms presenting the classification of straight lines in the skyline are shown in Fig. 12 (b). The number of categories has been set to five, unlike the width of each category which is variable and depends on parameters extracted from the processed photo. The zoom on the colorful skyline resulted from the curvature analysis is shown in Fig. 12 (c) for the different landscapes. After the curvature analysis, the CSS maps are depicted in the Fig. 12 (d) to describe the evolution of skyline points across the increasing scales of smoothing. The scatter plots in Fig. 12(e), as mentioned before, are made in order to visualize the evolution in the number of the skyline key points all over the different scales. These plotted curves prove the short lifetime of the key points across the scales in the natural landscapes, in the contrary, the points in urban landscapes persist until high scales.

\section{CONCLUSIONS AND FUTURE WORK}

In this paper, we aimed to introduce a new approach for the processing of landscapes' photos based on the skyline. Besides its usability in different applications, the horizon line, once extracted, is the same in different weather and lighting conditions. So, if we do extract useful information from the skyline, we can minimize the energy spent for searching information otherwise and overpass some problems like: luminosity, ambiguous colors or textures. Extracting some geometric features from the skyline was our first step to analyze this curve. The straight lines' classification gave an idea about the whole skyline and especially if the background in a landscape is almost natural or artificial. To enhance this classification, we have used the curvature scale space descriptor which analyze the skyline point per point.

Our work with the curvature descriptor should be expanded by making a tracking algorithm for some key points in the skyline. Thus, we will try to answer some questions like: Until which scale the buildings' corners still appear? Which points disappear from very low scale levels? The measurements picked from landscapes using the geometrical description will be the entry data for a classification algorithm. The results obtained using our skyline-based approach to identify natural scenes will be compared with similar works in the literature through a future work. 


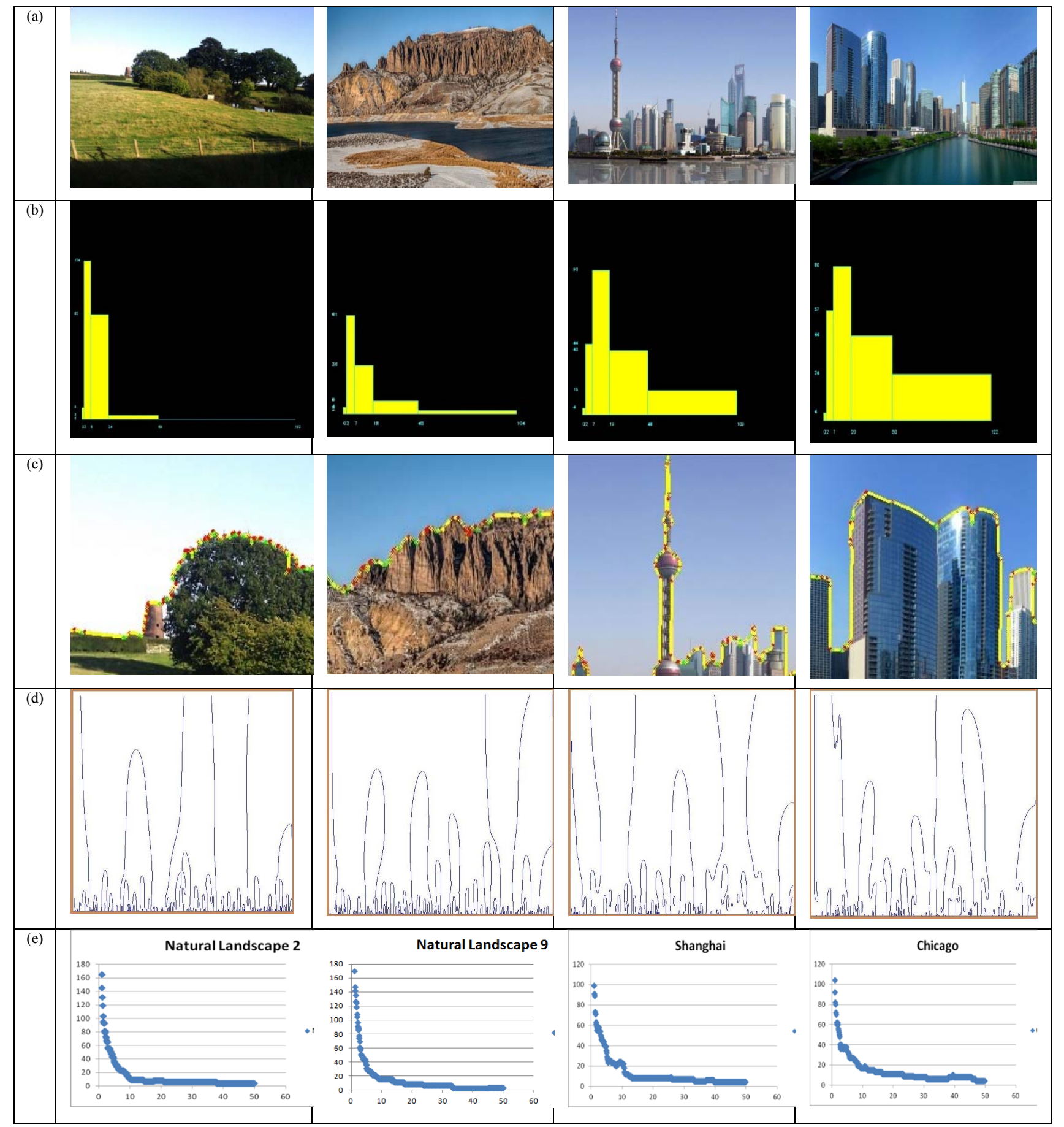

Fig. 12. Experimental results for different landscapes

(a) Landscapes photos

(b) Histograms of classification of straight lines

(c) The colorful skylines

(d) The CSS maps

(e) The evolution of skyline's key points in scales 


\section{ACKNOWLEDGEMENTS}

The authors would like to acknowledge the financial support of this work by grants from General Direction of Scientific Research (DGRST), Tunisia, under the ARUB program.

This work was initiated in the project SKYLINE which is led by EVS (Lyon2), with the following participants: l'EIVPVille de Paris, le LIRIS (Lyon1-2) and "l'Agence d'Urbanisme de Lyon."

\section{REFERENCES}

[1] J.C. Bazin, I.S. Kweon, C. Demonceaux, and P. Vasseur, “ Dynamic programming and skyline extraction in catadioptric infrared images," in 2009 IEEE International Conference on Robotics and Automation Kobe, Kobe, Japan, May 12-17, 2009.

[2] J.H. Woo, I.S. Kweon, G.S. Kim, and I.C. Kim, "Robust horizon and peak extraction for vision-based navigation," MVA2005 IAPR Conference on Machine Vision Applications, Tsukuba Science City, Japan, pp. 526-529, May 16-18, 2005.

[3] E. Gershikov, T. Libe, and S. Kosolapov, "Horizon line detection in marine images: which method to choose? ," International Journal on Advances in Intelligent Systems, vol 6 no 1 \& 2, 2013.

[4] T. Stone, M. Mangan, P. Ardin, and B. Webb, "Sky segmentation with ultraviolet images can be used for navigation," in Proceedings Robotics: Science and Systems, 2014.
[5] S. Ramalingam, S. Bouaziz, P. Sturm, and M. Brand, "Geo-localization using skylines from omni-images," In S3DV, 2009.

[6] M. Bansal and K. Daniilidis, "Geometric urban geolocalization," in Computer Vision and Pattern Recognition (CVPR), pp. 3978- 3985, 2014.

[7] G. Baatz , O. Saurer , K. Köser and M. Pollefeys, "Large scale visual geo-localization of images in mountainous terrain," Proceedings of the 12 th European conference on Computer Vision, Florence, Italy, October 07-13, 2012.

[8] J. Zhu, M. Bansal, N. V. Valk, and H. Cheng, "Adaptive rendering for large-scale skyline characterization and matching," in Proceedings 12th European Conference on Computer Vision Workshops and Demonstrations, Part I, pp. 163-174, Florence, Italy, October 2012.

[9] W. Liu, and C. Su, "Automatic peak recognition for mountain images," in Advanced Technologies, Embedded and Multimedia for Humancentric Computing (EMC 2013), Taipei, Taiwan, August 23-25, 2013.

[10] F. Mokhtarian, and A.K. Mackworth, "A Scale-based description and recognition of planar curves and two-dimensional shapes," in IEEE Transactions on Pattern Analysis and Machine intelligence, vol 8, no 1 pp.34-43, 1986.

[11] F. Mokhtarian, and S. Abbasi, "Shape similarity under affine transforms," The journal of Pattern Recognition 35, pp. 31 - 41, 2002.

[12] F. Mokhtarian, and R. Suomela, "Robust image corner detection through curvature scale space," in IEEE Transactions on Pattern Analysis and Machine intelligence, vol 20, no 12, December 1998. 